\title{
Comparison of Malaria Rapid Diagnostic Test Kit and Microscopy
}

\author{
Oluwakemi Bukola Runmonkun ${ }^{1,2}$, Omotayo Oluranti Ebong ${ }^{1,3}$, Udeme Owunari Georgewill ${ }^{1}$ \\ ${ }^{1}$ Department of Pharmacology, Faculty of Basic Medical Sciences, College of Health Sciences, University of Port Harcourt, \\ Port Harcourt, Nigeria \\ ${ }^{2}$ Pharmaceutical Society of Nigeria, Abuja, Nigeria \\ ${ }^{3}$ West African Society for Pharmacology, Abuja, Nigeria \\ Email: ayaarunmonkun@yahoo.com
}

How to cite this paper: Runmonkun, O.B., Ebong, O.O. and Georgewill, U.O. (2019) Comparison of Malaria Rapid Diagnostic Test Kit and Microscopy. Pharmacology \& Pharmacy, 10, 109-115.

https://doi.org/10.4236/pp.2019.103009

Received: January 21, 2019

Accepted: March 16, 2019

Published: March 19, 2019

Copyright $\odot 2019$ by author(s) and Scientific Research Publishing Inc. This work is licensed under the Creative Commons Attribution International License (CC BY 4.0).

http://creativecommons.org/licenses/by/4.0/ (c) (i) Open Access

\begin{abstract}
Malaria is the most common single diagnosis made in many countries in Africa. Microscopy is the gold standard for laboratory diagnosis of malaria parasite, but it requires adequate training and the time to get results is longer than that for Rapid Diagnostic Tests (RDTs). Use of RDTs is an alternative diagnostic method. This method is quick and easy to carry out. This study examined 412 blood samples of patients who met the study inclusion criteria from the health centres. All of the samples obtained were tested by SD Bioline malaria Ag P.f test commonly available in Nigeria and parasite count was done from thick film prepared. The results obtained were presented as a proportion positive in comparison to the total number of participants enrolled. Of the 412 enrollees, 284 were tested positive with malaria using the RDT, 400 were tested positive with microscopy and 12 were negative using microscopy and RDT. This study confirms the superior sensitivity of microscopy to RDTs in diagnosis of malaria. Although RDTs are very useful for quick diagnosis of malaria, particularly in areas where access to the use of microscopy is not available, the possibility of a low performance by RDTs in malaria diagnosis should be emphasized on health practitioners and microcopy should be encouraged as much as possible.
\end{abstract}

\section{Keywords}

Microscopy, Rapid Diagnostic Test, Malaria, Malaria Diagnosis

\section{Introduction}

Presumptive antimalarial treatment for any fever with no obvious alternative 
cause is widely practiced, and studies suggest that this leads to significant overuse of antimalarial drugs throughout [1] [2].

A majority of such cases who go on to treat for malaria may not be infected with plasmodium parasite. Diagnosis of malaria should be prompt and subsequent treatment should be immediate to prevent transmission of the disease. Microscopy, rapid diagnostic test, molecular methods, symptomatic method and serology are used for malaria diagnosis. Symptomatic diagnosis is often used because it is convenient but may lead to erroneous treatment because not all febrile cases presented have a corresponding positive plasmodium parasitemia. Microscopy remains the gold standard for diagnosis of malaria [3]. It is the mainstay of malaria diagnosis in most standard, well equipped health clinics and hospitals but the quality of microscopy-based diagnosis is frequently inadequate [4]. As useful as the use of microscopy in diagnosis may be, not all hospitals are equipped with a functional microscope and there may be inaccuracy in the results obtained due to unskilled technician or badly prepared slides.

A rapid diagnostic test (RDT) kit is a device that detects malaria antigen in a small amount of blood, usually $5-15 \mu \mathrm{L}$, by immunochromatographic assay with monoclonal antibodies impregnated on a test strip directed against the target parasite antigen. RDTs require no electricity, are easy to perform, and are cheap. It requires no skill and the results are obtained almost immediately. The results are seen in about 12 minutes [5].

The use of microscopy for diagnosis on the other hand, although accurate, requires training and is also time consuming. The slides need to be properly prepared and also read accurately.

The objective of this study is to compare the sensitivity of one commonly used RDT in Nigeria with microscopy as the standard for prompt and accurate diagnosis of malaria.

\section{Methods}

Study Site: This study was carried out in two Primary Health Centres in Port Harcourt, Rivers State, Nigeria from August to September 2017. These facilities cater for surrounding communities. Port Harcourt city is host to many multi-national oil companies, and as such the population is a mix of indigenes and non-indigenes of different tribes and ethnic groups. The city is located in the south-south region of Nigeria within the estuaries of River Niger and close to the Atlantic Ocean. The presence of mangrove forests, tropical rain forest and freshwater swamps that make up the vegetation in the area in addition to incessant rainfall year round and high humidity, breeds mosquitoes easily and malaria transmission occurs throughout the year.

\section{Recruitment Procedures}

Ethical clearance: Ethical clearance was obtained from the Research Ethics committee of the University of Port Harcourt and informed consent was sought and obtained from the parent or caregiver. 


\section{Inclusion and Exclusion Criteria}

These include the number of parasites must be between 1000 and $10,000 / \mu l$, axillary temperature of $\geq 37.5^{\circ} \mathrm{C}$, age between 6 and 59 months, ability to swallow, absence of febrile condition or severe malnutrition and ability and willingness to see the study through for the duration of the study.

\section{Sample Size}

This was calculated using the formula

$$
n=Z^{2} P(1-P) / d^{2}
$$

where

$$
\begin{aligned}
& P=\text { Prevalence of target group to have malaria; } \\
& Z=\mathrm{Z} \text { statistics at a given level of confidence; } \\
& d=\text { margin of error. }
\end{aligned}
$$

\section{Procedure}

Blood was collected into EDTA bottles from a total of 412 children aged between 6 to 59 months who met the study inclusion criteria. Thick films were prepared on slides in duplicates. The slides were stained with Field stain A and B. A drop of oil immersion was dropped on the slide which was then viewed under the microscope using $100 \times$ objective. The parasites were counted using a Tally counter. Counting was stopped once the number of parasite counted was over 100 in 200 WBCs or less than 100 in 500 WBCs. The actual number of parasites and white blood cells counted were recorded and used to calculate the parasite densities. Due to frequent lack of facilities in some malaria-endemic countries, in order to quantify WBCs of patients, an assumed WBC of $8.0 \times 10^{9} / 1$ has been set by World Health Organization to help in estimating malaria parasite densities [6]. The parasite density was calculated using the formula

$$
\text { Parasites } / \mu \mathrm{l} \text { of blood }=\frac{\text { Number of parasite counted } \times 8000 \text { white blood cells } / \mu \mathrm{l}}{\text { Number of white blood cells counted }}
$$

Rapid diagnostic test was carried out using SD malaria test kit. SD BIOLINE malaria Ag P.f test is a rapid and qualitative test for the detection of histidine-rich protein II (HRP-II) antigen of Plasmodium falciparum species in human blood. Four drops of blood was placed at the round specimen well using a capillary pipette. Then 4 drops of the assay diluent was added to the well. The results were read after a wait of 15 minutes. The results were negative if there was only one line ("C") in the result window, it was positive if 2 lines ("C"; "Pf") were present or invalid if there was no line ("C") in the result window.

Malaria cases that reports in these health centres vary between 10 - 15 patients seen per day of which the required age of 6 - 59 months is about $60 \%$ of the attendees. The body temperature of the enrollees must be above $37^{\circ} \mathrm{C}$. The test kit used must be SD Bioline and no other RDT. The test results must be confirmed by two technicians. All samples were first analyzed by RDT then by microscopy.

\section{Results}

A total of 412 patients aged between 6 to 59 months (mean $27.8 \pm 12.7$ months) 
suspected of malaria were recruited in the study. The results show that there were more males $(53.39 \%)$ than females $(46.60 \%)$ and the average weight was $13.2 \pm 2.7 \mathrm{~kg}$ (Table 1). All participants presented with fever $\left(37.9^{\circ} \mathrm{C} \pm 1.04^{\circ} \mathrm{C}\right)$.

All the participants enrolled were first tested with RDT followed by microscopy. Of the 412 participants enrolled, 284 (68.93\%) tested positive with malaria using RDT, 400 (97.08\%) tested positive with microscopy. Twelve $(2.91 \%)$ of the RDT negative were also negative with microscopy. All those who tested positive with RDT were also positive with microscopy (Table 2).

\section{Discussion}

Malaria diagnosis should be prompt, followed by immediate and appropriate treatment to prevent transmission of the disease and development of complicated malaria. Malaria should be regarded as a potential medical emergency and treated accordingly. Delays in diagnosis and treatment will lead to increased mortality and morbidity [7].

Symptomatic diagnosis also referred to as clinical diagnosis, is often used because it is convenient but may lead to erroneous treatment because not all febrile cases presented have a corresponding positive plasmodium parasitemia. In our study, $12(2.91 \%)$ participants had negative results using both microscopy and RDT.

WHO recommends prompt malaria diagnosis either by microscopy or malaria rapid diagnostic test (RDT) in all patients where malaria is suspected before administering treatment with antimalarials. Diagnostic testing improves the overall management of patients with febrile illnesses, and it may also help to reduce the emergence and spread of drug resistance by reserving treatment with antimalarials for those who actually have the disease. In as much as microscopy plays an important role in diagnosis of malaria, a major disadvantage is that it

Table 1. Demographics of participants.

\begin{tabular}{ccc}
\hline Demographics & & Number (\%) \\
\hline Gender & Male & $220(53.39 \%)$ \\
& Female & $192(46.60 \%)$ \\
Temperature $\left({ }^{\circ} \mathrm{C}\right)$ & & $37.9 \pm 1.04$ \\
Weight $(\mathrm{kg})$ & $13.2 \pm 2.7$ \\
Age & 6 - 59 month & $100 \%$ \\
\hline
\end{tabular}

Table 2. Comparison of diagnostic test results.

\begin{tabular}{ccc}
\hline & Microscopy & RDT \\
\hline Total number & 412 & 412 \\
Positive & 400 & 284 \\
Negative & 12 & 128 \\
Percentage of positive & $97.08 \%$ & $68.93 \%$ \\
\hline
\end{tabular}


requires a level of skill that is often not available in many health facilities in countries where malaria is endemic. There may also be flaws in the preparation of the slides and in the reading of slides.

In this study, we assessed the performance of SD Bioline test kit and the use of microscopy using Field stain A and B thick blood films. We found that SD Bioline test kit had the lower test results compared to microscopy. It is possible that some malaria infections detected by blood films were not detected by SD Bioline. Another possibility is that some of the participants may have been medicated when symptoms appeared initially and provided no information of such at the health centres. Other reasons for the low positive rates may be due to other factors that could affect the stability of the RDT like extreme temperatures that may affect the efficacy of the RDT which may occur during storage and transportation [8]. High humidity has also been reported to degrade RDTs [9] and this may be an explanation for the low positive results as Port Harcourt is highly humid.

This study has shown that SD Bioline Malaria Ag P.f is easy to use and the results were ready in 12 minutes. The ability of this RDT to detect parasite antigens (Histidine Rich Protein-II) from finger prick blood, allowing efficient handling for use by non-technician with less training [10] is bolstered by our findings.

The use of microscopy however, gave better (97.08\%) results than use of RDT. These results are similar to those obtained by Azikwe et al. (2012) [11], Harcut et al. (2013) [12] and Elechi et al. (2015) [13].

Another batch of SD Bioline or another type of RDT may be more effective than the batch used. So there is need to investigate further other RDTs in the Nigerian market in order to conclude on the efficacy of use of RDTs in Nigeria in malaria diagnosis but it is important to draw the attention of health workers to the performance of reliance only on RDT because as shown in this study, a positive case may have been missed.

\section{Limitations}

Delays in transportation can affect the sensitivity of the RDTs as the storage conditions on transportation may not be optimum. One of the requirements of storage is that the RDTs are stored under cool conditions. Due to the climatic condition in the country and irregularity of power supply, the storage conditions of the RDTs may have been compromised. The use of only one batch of RDT may also affect the results. Another batch may have performed better. There may be a need to use more than one batch and type of RDT to conclude on the sensitivity of RDTs.

\section{Conclusion}

This study confirms that microscopy is more accurate in diagnosis of malaria. It is important to note that a negative malaria RDT results may not necessarily in- 
dicate absence of malaria. We recommend the use of microscopy as much as possible to be done after the use of RDT for malaria diagnosis.

\section{Acknowledgements}

We wish to acknowledge $1^{\text {st }}$ Sylvanus J. S. Cookey (Professor of Malaria study), Dr. Cecelia Nwibubasa, Dr. Okara, Mrs. Vivian Agi, Mrs Kalainne Banigo, and the technical staff of the medical laboratories at Mile 1 and Orogbum Health Centres.

\section{Financial Support}

None.

\section{Conflicts of Interest}

We declare no conflict of interest.

\section{References}

[1] Barat, L., Chipipa, J., Kolczak, M. and Sukwa, T. (1999) Does the Availability of Blood Slide Microscopy for Malaria at Health Centers Improve the Management of Persons with Fever in Zambia? The American Journal of Tropical Medicine and Hygiene, 60, 1024-1030. https://doi.org/10.4269/ajtmh.1999.60.1024

[2] Amexo, M., Tolhurst, R., Barnish, G. and Bates, I. (2004) Malaria Misdiagnosis: Effects on the Poor and Vulnerable. The Lancet, 364, 1896-1898.

https://doi.org/10.1016/S0140-6736(04)17446-1

[3] WHO (2010) Guidelines for the Treatment of Malaria. 2nd Edition, WHO, Geneva. http://www,who.int/mediacentre/news/reiease/2010/malaria_20100308/en/

[4] World Health Organization (2015) World Malaria Report. WHO, Geneva.

[5] Moody, A. (2002) Rapid Diagnostic Tests for Malaria Parasites. Clinical Microbiology Reviews, 15, 66-78. https://doi.org/10.1128/CMR.15.1.66-78.2002

[6] Adu-Gyasi, D., Adams, M., Amoako, S., Mahama, E., Nsoh, M., Amenga-Etego, S., Baiden, F., Asante, K.P., Newton, S. and Owusu-Agyei. S. (2012) Estimating Malaria Parasite Density: Assumed White Blood Cell Count of 10,000/ $\mu$ l of Blood Is Appropriate Measure in Central Ghana. Malaria Journal, 11, 238. https://doi.org/10.1186/1475-2875-11-238

[7] Kain, K.C., Harrington, M.A., Tennyson, S. and Keytone, J.S. (1998) Imported Malaria; Prospective Analysis of Problems in Diagnosis and Management. Clinical Infective Disease, 292, 721-723. https://doi.org/10.1086/514616

[8] Jorgensen, P., Chanthap, L., Rebueno, A., Tsuyuoka, R. and Bell, D. (2006) Malaria Rapid Diagnostic Tests in Tropical Climates: The Need for a Cool Chain. The American Journal of Tropical Medicine and Hygiene, 74, 750-754. https://doi.org/10.4269/ajtmh.2006.74.750

[9] Abanyie, F.A., Arguin, P.M. and Gutman, J. (2011) State of Malaria Diagnostic Testing at Clinical Laboratories in the United States, 2010: A Nationwide Survey. Malaria Journal, 10, 340. https://doi.org/10.1186/1475-2875-10-340

[10] Geoffrey, P.E. and John, W. (2002) Evaluation of the ICT Malaria P.f/P.v and the Optimal Rapid Diagnostic Tests for Malaria in Febrile Returned Travelers. Journal of Clinical Microbiology, 40, 4166-4171. 
https://doi.org/10.1128/JCM.40.11.4166-4171.2002

[11] Azikiwe, C.C.A., Ifezulike, C.C., Siminialayi, I.M., Amazu, L.U., Enye, J.C. and Nwakwunite, O.E. (2012) A Comparative Laboratory Diagnosis of Malaria: Microscopy versus Rapid Diagnostic Test Kits. Asian Pacific Journal of Tropical Biomedicine, 2, 307-310. https://doi.org/10.1016/S2221-1691(12)60029-X

[12] Harchut, K., Stanley, C., Dobson, A., Klaassen, B., Rambaud-Aithaus, C. and Althaus, F. (2013) Over-Diagnosis of Malaria by Microscopy in the Kilombero Valley, Southern Tanzania: An Evaluation of the Utility and Cost-Effectiveness of Rapid Diagnostic Tests. Malaria Journal, 12, 159.

https://doi.org/10.1186/1475-2875-12-159

[13] Elechi, H.A, Rabasa, A.L., Bashir, M.F., Gofama, M.M., Ibrahim, H.A. and Askira, U.M. (2015) Uncomplicated Malaria in Children: The Place of Rapid Diagnostic Test. Nigerian Medical Journal, 56, 85-90.

https://doi.org/10.4103/0300-1652.150686 